\title{
Gauging Cardiac Repair and Regeneration with New Molecular Probes
}

\author{
James T. Thackeray and Frank M. Bengel
}

Department of Nuclear Medicine, Hannover Medical School, Hannover, Germany

$\mathbf{R}$ tive of cell-based therapy over the last decade. The first generation of clinical stem cell trials in cardiology, however, have borne only modest success, such that the regeneration of mature cardiomyocytes remains elusive (1). Rather, the observed benefits are ascribed to enhanced damage repair via paracrine mechanisms and endogenous cell recruitment. Accordingly, regenerative cardiology has begun to move away from transplantation of exogenous stem cells, rather aiming to support and enhance the natural healing process (2). As such, infarct healing and the cellular mechanisms underlying this process have emerged as a target for novel molecular imaging probes.

Infarct healing is a complex and multifaceted process that offers several molecular targets for therapy and imaging. After myocardial infarction, there is an immediate and organized infiltration of inflammatory leukocytes, which enact a series of both protective and adverse consequences to the damaged myocardium. The protective role of inflammatory leukocytes lies in the engulfment and removal of dead and dying cells, and isolation of cellular damage from the healthy myocardium, allowing for the generation of stable, collagen-rich scar tissue. Invading leukocytes also secrete chemotactic factors to recruit a secondary wave of more reparative inflammatory cell types, including M2-like macrophages, some lymphocytes, and a small proportion of bone marrow-derived progenitor cells, which contribute to angiogenesis and cardioprotection (Fig. 1A). The inflammatory cascade can be detrimental, however, as it is believed that the early wave of granulocytes, lymphocyte antigen 6 complex ${ }^{\text {high }}$ (Ly6C $\mathrm{C}^{\text {high }}$ ) monocytes, and differentiated M1-like macrophages perpetuates inflammation and contributes to infarct expansion, leading to higher degrees of remodeling $(3,4)$. Striking a balance between leukocyte-mediated healing and rigorous inflammation is optimal but can be complicated in numerous ways (Figs. 1B-1E). Exacerbated proinflammatory cell activity leads to scar instability and left ventricular rupture in mice, which is thought to manifest as infarct expansion in humans. A prolonged proinflammatory response may increase infarct size and contribute to worse remodeling. Shifting proportions of leukocyte subpopulations, including altered granulocyte content or decreased reparative cell recruitment, can have deleterious effects on cardiac function. Complete early suppression of inflammation increases the rate of left ventricular rupture and late remodeling. Novel

Received Nov. 29, 2017; revision accepted Dec. 14, 2017.

For correspondence or reprints contact: James T. Thackeray, Hannover Medical School, Carl Neuberg Strasse 1, Hannover, 30625 Germany.

E-mail: thackeray.james@mh-hannover.de

Published online Jan. 11, 2018.

COPYRIGHT (c) 2018 by the Society of Nuclear Medicine and Molecular Imaging. DOI: 10.2967/jnumed.117.198283 treatment strategies seeking to augment the protective mechanisms while dampening the maladaptations can be challenging because the substrate is constantly changing and comprised of a diverse collection

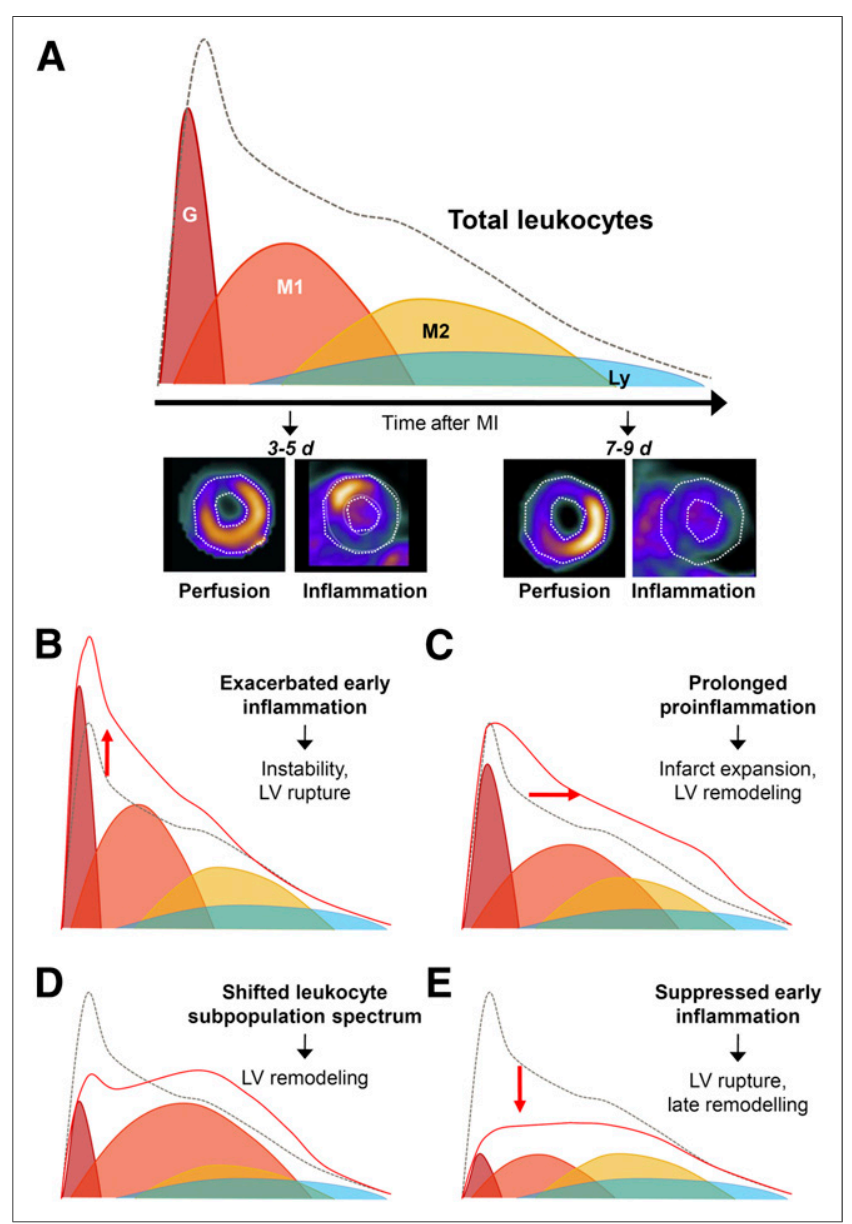

FIGURE 1. Dynamic infiltration of inflammatory leukocytes into infarct territory after ischemia. (A) Under normal conditions, initial wave of granulocytes $(G)$ begins to extravasate within hours of insulin and is followed by proinflammatory Ly6Chigh monocytes and M1-like macrophages (M1), which are maximal at 3-5 d after myocardial infarction. Reparative M2-like macrophages (M2) predominate at later stages, 7-9 d after myocardial infarction, and lower levels of lymphocytes (Ly). Imaging with ${ }^{68} \mathrm{Ga}$-pentixafor for C-X-C chemokine receptor type 4 shows accumulation in perfusion defect early (3-5 d) but not at later stages (7-9 d) after infarction. Total leukocyte presence is described by dashed integral line. Infarct healing and cardiac repair can be affected by altered dynamics or intensity of leukocyte infiltration (red integral), including elevated early proinflammatory cell infiltration (B), delayed clearance of proinflammatory cells $(C)$, shifted proportions of leukocyte subtypes (D), or complete suppression of early inflammation (E). LV = left ventricular; $\mathrm{MI}=$ myocardial infarction. 
of inflammatory and noninflammatory cell subpopulations, such that it is difficult to determine the optimal time for effective therapy. It is here that molecular imaging may play an invaluable role, but understanding the substrate of individual imaging markers is essential.

Direct interrogation of infiltrating immune cells, which define the infarct healing process, may provide insight into the progression of disease and response to therapy. Predominantly, immune cell imaging has relied on the enhanced metabolism of activated leukocytes, particularly proinflammatory M1-like macrophages (5). Emerging evidence suggests that the early postmyocardial infarction ${ }^{18} \mathrm{~F}-\mathrm{FDG}$ signal may predict subsequent function decline (6). Interpretation of these images is complicated by the mixed substrate, wherein residual viable but ischemically compromised (and thus not suppressible) myocytes may contribute to the total tracer signal (7). Accordingly, novel molecular imaging probes with limited or no cardiomyocyte background signal have been proposed to target more specific leukocyte subpopulations, including ${ }^{68} \mathrm{Ga}$-pentixafor for C-X-C chemokine receptor type 4 (8) and ${ }^{18} \mathrm{~F}$-flutriciclamide (GE180) for mitochondrial translocator protein (9). The potential value of specific imaging markers is found in enabling more intricate dissection of the inflammatory cascade (and subsequent repair), but the relationship to long-term outcome remains to be elucidated.

Repair-targeted molecular imaging may provide an avenue for streamlining of clinical cardiovascular trials using novel inflammation-directed drugs (10). Evaluation of novel targeted therapies may be enhanced by concurrent imaging of the repair mechanisms $(11,12)$, which in turn may help to identify appropriate patient populations and subpopulations for the intervention.

Just as regenerative medicine has evolved from basic cell transplantation to reprogramming of endogenous mechanisms to support repair, so too must molecular imaging adapt to provide the requisite tools to evaluate the molecular mechanisms underlying endogenous healing. Although novel molecular probes have been developed, it will be essential to delineate the specific cell populations targeted by these compounds to discern their added value in patient care. Indeed, the goal for molecular imaging in cardiac repair should ultimately extend not only to identification of therapeutic targets but also to selection of optimal patients and to appropriate timing of intervention to maximize therapeutic benefit. In doing so, we may identify the correct treatment for the correct patient at the correct time.

\section{DISCLOSURE}

No potential conflict of interest relevant to this article was reported.

\section{REFERENCES}

1. Mathur A, Fernandez-Aviles F, Dimmeler S, et al. The consensus of the task force of the European Society of Cardiology concerning the clinical investigation of the use of autologous adult stem cells for the treatment of acute myocardial infarction and heart failure: update 2016. Eur Heart J. 2017;38:2930-2935.

2. Kovacic JC, Fuster V. Cell therapy for patients with acute myocardial infarction: accrued evidence to date. Circ Res. 2015;116:1287-1290.

3. Swirski FK, Nahrendorf M. Leukocyte behavior in atherosclerosis, myocardial infarction, and heart failure. Science. 2013;339:161-166.

4. Jung K, Kim P, Leuschner F, et al. Endoscopic time-lapse imaging of immune cells in infarcted mouse hearts. Circ Res. 2013;112:891-899.

5. Lee WW, Marinelli B, van der Laan AM, et al. PET/MRI of inflammation in myocardial infarction. J Am Coll Cardiol. 2012;59:153-163.

6. Rischpler C, Dirschinger RJ, Nekolla SG, et al. Prospective evaluation of ${ }^{18} \mathrm{~F}-$ fluorodeoxyglucose uptake in postischemic myocardium by simultaneous positron emission tomography/magnetic resonance imaging as a prognostic marker of functional outcome. Circ Cardiovasc Imaging. 2016;9:e004316.

7. Thackeray JT, Bankstahl JP, Wang Y, Wollert KC, Bengel FM. Clinically relevant strategies for lowering cardiomyocyte glucose uptake for ${ }^{18} \mathrm{~F}-\mathrm{FDG}$ imaging of myocardial inflammation in mice. Eur J Nucl Med Mol Imaging. 2015;42:771-780.

8. Thackeray JT, Derlin T, Haghikia A, et al. Molecular imaging of the chemokine receptor cxcr4 after acute myocardial infarction. JACC Cardiovasc Imaging. 2015;8:1417-1426.

9. Thackeray JT, Hupe HC, Wang Y, et al. Molecular imaging of the heart-brain axis: post-infarct myocardial inflammation predicts subsequent remodeling and triggers neuroinflammation. J Am Coll Cardiol. 2018;71:263-275.

10. Nahrendorf M, Frantz S, Swirski FK, et al. Imaging systemic inflammatory networks in ischemic heart disease. J Am Coll Cardiol. 2015;65:1583-1591.

11. Jujo K, Hamada H, Iwakura A, et al. Cxcr4 blockade augments bone marrow progenitor cell recruitment to the neovasculature and reduces mortality after myocardial infarction. Proc Natl Acad Sci USA. 2010;107:11008-11013.

12. Ridker PM, MacFadyen JG, Everett BM, et al. Relationship of c-reactive protein reduction to cardiovascular event reduction following treatment with canakinumab: a secondary analysis from the cantos randomised controlled trial. Lancet. November 13, 2017 [Epub ahead of print]. 